\title{
terminal Terminal
}

Technologie de l'information, culture \& société

$118 \mid 2016$

Contrôle social, surveillance et dispositifs numériques

\section{Lanceurs d'alerte : vers un statut unifié?}

\section{Dominique Desbois}

revues.org

Édition électronique

URL : http://terminal.revues.org/1389

ISSN : 2429-4578

\section{Éditeur \\ CREIS-Terminal}

Ce document vous est offert par Institut des sciences et industries du vivant et de l'environnement - AgroParisTech

\section{AgroParisTech}

Référence électronique

Dominique Desbois, «Lanceurs d'alerte : vers un statut unifié ? », Terminal [En ligne], 118| 2016, mis en ligne le 15 octobre 2016, consulté le 23 mai 2017. URL : http://terminal.revues.org/1389

Ce document a été généré automatiquement le 23 mai 2017.

tous droits réservés 


\title{
Lanceurs d'alerte : vers un statut unifié ?
}

\author{
Dominique Desbois
}

Cousin français du whistleblower anglo-saxon, le terme de "lanceur d'alerte », a été popularisé par la publication en 1999 de l'ouvrage des sociologues Francis Chateauraynaud et Didier Torny intitulé "Les sombres précurseurs", vulgarisant les recherches menées dans le cadre du programme CNRS « Risques collectifs et situations de crise » coordonné par Claude Gilbert. Chimiste toxicologue responsable d'un programme de recherche sur les éthers de glycols, André Cicolella, licencié en 1994 par l'Institut national de recherches et de sécurité, a également contribué par son combat juridique à faire connaître la problématique du lanceur d'alerte en fondant le Réseau Environnement Santé (interdiction du bisphénol $\mathrm{A}$ dans les biberons et du perchloroéthylène pour le nettoyage à sec); avec le député vert André Aschieri, il est également à l'origine de la création de l'Agence française de sécurité sanitaire. Nombreux, sont celles et ceux comme Henri Pézeart, toxicologue du CNRS et animateur du comité anti-amiante de Jussieu, ou Irène Frachon, pneumologue du $\mathrm{CHU}$ de Brest ayant révélé la nocivité cardiaque du bentfluorex (Mediator), qui ont affronté de puissant lobbys au risque de leur réputation pour dénoncer des atteintes graves à l'environnement ou à la santé.

\section{Sombres traîtres ou perdants magnifiques?}

2 Parmi cette galerie de portraits figurent désormais les «Don Quichotte » du numérique tels Chelsea Mannings, condamnée à 35 ans de prison pour avoir transmis en juillet 2010 les war-logs de la guerre en Afghanistan, Julian Assange, fondateur de Wikileaks réfugié à l'Ambassade d'Équateur de Londres depuis juin 2012 pour échapper à un mandat d'arrêt américain, et Edward Snowden qui, ayant révélé les écoutes électroniques de la NSA, a dû demander l'asile en Russie pour échapper aux poursuites au titre de l'Espionage Act utilisé pas moins de sept fois par l'administration américaine au cours des deux mandats du Président Obama. 
$3 \mathrm{Au}$ cours de cette lutte du pot de terre contre le pot de fer, la protection juridique des lanceurs d'alerte a progressé : désormais, une soixantaine de pays ont promulgué des dispositions législatives protégeant les lanceurs d'alerte et sept d'entre eux peuvent être considérés comme offrant une protection significative contre les menaces ou représailles. Parmi les pays de l'OCDE, ceux ayant adopté une protection légale spécifique des lanceurs d'alerte sont l'Australie, la Belgique, le Canada, la Corée du Sud, la Hongrie, le Japon, la Nouvelle Zélande, les Pays-Bas, le Royaume-Uni et les USA.

4 Dans la dynamique de mobilisation suscitée par des associations anti-corruption comme Transparency International, antiCOR ou la Plate-forme internationale des lanceurs d'alerte (PILA), la reconnaissance d'un statut cohérent et unifié du «lanceur d'alerte » constitue une étape importante dans la lutte contre des délits transnationaux à laquelle contribuent de manière irremplaçable les lanceurs d'alerte, le plus souvent à leurs risques et périls.

5 En témoigne, la récente affaire «Luxleaks » où Antoine Deltour ${ }^{1}$, employé par le cabinet de consultants PricewaterhouseCooper, a été inculpé pour avoir transmis au journaliste Édouard Perrin (émission Cash Investigation de France 2) des "rescrits fiscaux» confidentiels de l'administration du Grand-Duché mettant en lumière des accords anticipés («tax rulings ») permettant aux multinationales de se soustraire à l'imposition. Pour cette alerte, le procureur du Grand-Duché a requis dix-huit mois d'emprisonnement à l'encontre d'Antoine Delcourt, récipiendaire 2016 du prix Éthique décerné par l'association antiCOR. Le 29 juin, la justice luxembourgeoise a condamné Antoine Deltour à 12 mois de prison avec sursis et $1500 €$ d'amende. L'affaire sera jugée en appel du 30 novembre au 12 décembre à Luxembourg, alors que 108 des 751 députés européens ont exprimé le 8 septembre leur soutien à Antoine Deltour et Raphaël Halet, co-accusé, dans les procédures judiciaires en cours.

6 Cependant, sans garanties pour le lanceur d'alerte, les montages financiers extraterritoriaux révélés par la diffusion des documents panaméens (archives du cabinet Mossack Fonseca) couvrant une myriade d'opérations d'évasion fiscale et de blanchiments de fraude fiscale n'auraient jamais été mis au jour.

\section{Un repenti à 78 millions de dollars et une femme qui en savait trop}

7 Ainsi, le parcours de Stéphanie Gibaud, responsable du marketing événementiel chez UBS France, victime dans l'affaire "UBS », déclenchée initialement par les révélations de Bradley Birkenfeld en 2007 aux USA dévoilant les pratiques d'incitation à l'évasion fiscale de la banque suisse UBS, illustre également de façon exemplaire la nécessité de protéger les lanceurs d'alerte.

8 Condamnée à verser en 2009 une amende de 780 millions de dollars, la banque UBS s'est vue contrainte de transmettre au fisc américain l'identité de 4450 clients soupçonnés de l'avoir fraudé. Pour Bradley Birkenfeld, qui ne s'est opposé aux pratiques de son employeur qu'après avoir compris qu'il serait le seul inculpé lors de leur découverte, le repentir paye puisque, d'après le New York Times, il a perçu en récompense 78 millions de dollars net d'impôts.

9 Moins opportuniste, Stéphanie Gibaud résiste en juin 2008 à une demande hiérarchique de détruire les fichiers de prospects, clients et chargés d'affaires mettant en lumière 
l'incitation à l'évasion fiscale pratiquée par la banque UBS, demande effectuée suite à une perquisition du bureau de son Directeur général à Paris. Par la suite, elle se plaint de discriminations auprès de l'inspection du Travail, puis du Procureur de la République. En 2012, elle est entendue par Éric Boquet, rapporteur de la Commission Évasion Fiscale. Au terme d'une tentative de licenciement en 2009 et d'une plainte d'UBS à son encontre pour diffamation qui sera déboutée, Stéphanie Gibaud est licenciée par UBS en 2012. Depuis, elle n'a pas retrouvé de travail. Le 5 mars 2015, le tribunal des Prudhommes de Paris condamnait UBS pour harcèlement à son encontre.

\section{Une reconnaissance étendue}

10 Adopté en première lecture à l'Assemblée nationale par les députés, le projet de loi relatif à la transparence, à la lutte contre la corruption et à la modernisation de la vie économique (dit «Sapin II») prévoit des dispositions instituant une reconnaissance étendue du rôle des lanceurs d'alerte par la définition d'un statut leur assurant une meilleure protection.

11 En effet, lors de la première séance publique, les députés ont adopté lundi 6 juin vers minuit dans un hémicycle clairsemé la définition suivante :

« Un lanceur d'alerte est une personne qui révèle, dans l'intérêt général et de bonne foi, un crime ou un délit, un manquement grave à la loi ou au règlement, ou des faits présentant des risques ou des préjudices graves pour l'environnement, la santé ou la sécurité publiques, ou témoigne de tels agissements. Il exerce son droit d'alerte sans espoir d'avantage propre ni volonté de nuire à autrui. L'alerte ne saurait révéler quelque élément que ce soit relevant du secret de la défense nationale, du secret médical ou du secret professionnel applicable aux relations entre un avocat et son client. ».

Débattu pendant plus d'une heure, cet amendement résulte d'un compromis entre un groupe de députés souhaitant ajouter la notion d'intérêt général et le rapporteur Sébastien Denaja (Parti Socialiste) qui y était défavorable. Pour Michel Sapin, ministre des Finances et des Comptes publics :

«La volonté du Gouvernement c'est que toutes ces situations, et en particulier les situations qui concernent des lanceurs d'alerte qui, dans le domaine financier ou dans le domaine fiscal, auraient "lancé une alerte ", (...) que ces cas-là soient couverts. ».

\section{Une définition contestée}

Cependant, cette définition est contestée en l'état par PowerFoule, Transparency International France et la coordination des ONG qui militent pour une définition plus globale du lanceur d'alerte calquée sur les recommandations du Conseil de l'Europe, à l'instar de la proposition de loi déposée par le député Yann Galut (groupe Socialiste, Écologiste et Républicain, membre de la Gauche forte) :

«toute personne qui signale ou révèle, de bonne foi, une information relative à un crime, un délit, une menace ou un préjudice grave pour l'intérêt général, dont elle a la connaissance dans le contexte d'une relation de travail, rémunérée ou non, présente ou passée. ». lanceur d'alerte, pas moins de cinq textes de loi votés entre 2007 et 2013 contiennent des 
articles protégeant de façon sectorielle les signalements. À l'opposé du contexte national, plus d'une dizaine de pays dans le monde ont adopté une législation unifiée pour la protection des lanceurs d'alerte. Ainsi, selon Patrice Carvalho (député communiste), « notre droit actuel ne permet pas de garantir efficacement les lanceurs d'alerte ».

De fait, la protection des lanceurs d'alerte s'invite dans de multiples textes mais sans toutefois reposer sur un statut unique. Dernier exemple en date de ces avatars, le projet de «loi pour une République numérique » défendu par Axelle Lemaire, secrétaire d'État propose un statut des lanceurs d'alerte informatique concernant «des gens qui découvrent des failles informatiques et qui ne seront pas poursuivis pénalement s'ils les révèlent aux organisations concernées ». Seul amendement notable, le retrait de la mention du « secret des affaires » (adopté par 197 pour et 145 contre), anticipant sur les dispositions très contestées par les journalistes de la nouvelle directive européenne sur le secret d'affaires. Ainsi, l'observatoire des Libertés et du Numérique rassemblant plusieurs associations et syndicats regrette dans un communiqué de presse le décalage entre la débauche d'énergie suscitée par la consultation citoyenne et la portée limitée de ce texte, déplorant notamment «l'absence de dispositions plus protectrice des utilisateurs dans les chapitres de la loi traitant de la neutralité du Net ou des données personnelles » qui pourraient contribuer à la protection des lanceurs d'alerte à l'instar du développement d'un droit positif au chiffrement.

La sénatrice Nathalie Goulet (groupe des indépendants, membre de la Commission d'enquête sur l'évasion fiscale) réclame une uniformisation de leur statut juridique «la protection ou la législation pour les lanceurs d'alerte est construite en fonction des secteurs, et il y a des trous dans la raquette. Une partie des lanceurs d'alerte sont protégés contre le licenciement, d'autres non ». Pour l'une des lois les plus récentes (2013) concernant l'indépendance de l'expertise en matière de santé et d'environnement, la sénatrice Marie-Christine Blandin (groupe écologiste) dénonce le fait que «la loi de 2013 n'est pas appliquée ». Pourtant, c'est la seule loi en France qui autorise le recours aux média « pour tout salarié de bonne foi, témoin de crimes et délits dans l'exercice de son travail».

La recommandation du Conseil de l'Europe est qualifiée de «renforcement en demiteinte » du statut de lanceur d'alerte en raison de son caractère peu contraignant pour les États membres. Envisagée en 2010 par l'Assemblée parlementaire du Conseil de l'Europe, la solution plus contraignante d'une convention-cadre européenne a été abandonnée en raison des difficultés pour faire aboutir le processus de décision intergouvernementale. Toutefois, il semblerait possible d'aboutir à une directive européenne dans ce domaine dans un futur pas trop lointain.

Pour les organisations non-gouvernementales cosignataires de la pétition, il est nécessaire d'offrir des canaux de signalement clairs, accessibles et sécurisés (procédure hiérarchisée des trois-paliers : interne, juridico-administratif, médiatique), de garantir la confidentialité et si possible l'anonymat, de pouvoir maintenir le lanceur d'alerte dans son emploi, de réparer intégralement les préjudices physiques et moraux, et de prévoir des sanctions pénales à l'encontre des entraves au signalement et des représailles exercées. Enfin, ces ONG réclament la création d'une agence nationale de l'alerte qui soit véritablement indépendante. En effet, l'Agence française anti-corruption (AFA) prévue par l'article premier du projet de loi Sapin II, dépend des ministères du budget et de la justice, ce qui peut être préjudiciable par exemple à l'effectivité des poursuites dans les affaires de fraude fiscale et de blanchiment, l'administration conservant le privilège de 
l'opportunité des poursuites: ainsi, dans une complète opacité, la Commission des infractions fiscales ne transmets à la justice qu'environ un dossier sur dix.

\section{Au milieu du gué}

L'impératif n'est donc pas tant la célébration médiatique de quelques faits d'armes dans la lutte contre la corruption, le blanchiment, et la fraude fiscale qu'une réflexion plus approfondie et un engagement plus déterminé en faveur des conditions nécessaires pour que les citoyens en position de responsabilité sociétale puissent exercer un devoir d'alerte vis-à-vis de pratiques indignes nuisant à l'intérêt général dans une société solidaire et responsable. Dans cette vision démocratique d'un réel « contrôle social » de la gouvernance des firmes ou des administrations, qu'elles soient nationales ou transnationales, il nous semble toutefois que la notion "d'intérêt général », essentielle pour une couverture globale des catégories d'infraction, nécessite d'être sinon délimitée du moins déclinée dans chacun des champs d'application identifiés afin de ne pas laisser place à des interprétations soit très extensives qui pourraient nuire aux libertés individuelles (d'où l'exclusion du champ des informations soumis au secret médical ou professionnel) ou conduire à la dissémination incontrôlée d'informations sensibles (d'où l'exclusion des informations couvertes par le secret défense), soit à l'inverse trop restrictives qui pourraient interdire les poursuites et laisser perdurer des pratiques inadmissibles dans des " angles morts » tels que la défense ou le renseignement. En effet, à travers les quelques exemples présentés, la fiscalité, la santé, l'environnement apparaissent comme autant de domaines spécifiques où la lutte contre la fraude, la corruption, ou la collusion d'intérêts nécessitent une connaissance approfondie du champ d'application de la loi pour pouvoir par exemple distinguer entre un repenti, un « fuiteur d'informations" et un lanceur d'alerte ou qualifier les pratiques incriminées de frauduleuses ou d'abusives.

Ainsi, les tragédies personnelles vécues par les lanceurs d'alerte conduisent à réinterroger, dans les entreprises comme dans les administrations, les limites de la gouvernance en matière de responsabilité sociétale ou de libertés individuelles qui se trouvent sans cesse redéfinies par les nouvelles technologies, en particulier celles s'appuyant sur la numérisation de l'information, dans un contexte où les politiques fondées sur la terreur, le totalitarisme ou l'exclusion menacent la « société ouverte » de nos démocraties.

\section{NOTES}

1. Un comité de soutien à Antoine Deltour s'est constitué en 2015 (cf. https://supportantoine.org/asso/) : parmi ses objectifs, figurent l'harmonisation de la justice fiscale entre pays européens et la promotion de dispositifs de protection pour les lanceurs d'alerte. 


\section{AUTEUR}

DOMINIQUE DESBOIS 